\title{
An Egalitarian Argument against Reducing Deprivation
}

\author{
Julia Mosquera ${ }^{1,2}$
}

Accepted: 18 May 2017 / Published online: 2 December 2017

(C) The Author(s) 2017. This article is an open access publication

\begin{abstract}
Deprivations normally give rise to undeserved inequality. It is commonly thought that one way of improving a situation with respect to equality is by reducing the incidence of deprivations. In this paper I argue that there is at least one respect in which reducing the incidence of deprivations can make things worse from the point of view of equality. While eliminating deprivations leads to the elimination of inequalities, reducing the incidence of deprivations leads to an uneven distribution of the pairwise relations of inequality of a population, which leads to the concentration of pairwise relations of inequality in the worse off. If my argument is correct, egalitarians have reasons to broaden their dimensions of concern: egalitarians should not only be concerned about the unequal distribution of goods, but also about the unequal distribution of pairwise relations of inequality of a population.
\end{abstract}

Keywords Inequality $\cdot$ Deprivations $\cdot$ Egalitarianism $\cdot$ Population ethics $\cdot$ Larry Temkin

\section{The Paradox of Deprivation Reduction and Inequality}

The existence of deprivations often gives rise to undeserved inequality. It is an intuitive and common sense belief that reducing deprivations such as poverty leads to more equality. Indeed, many people who advocate poverty reduction describe themselves as egalitarians. This is indisputably true for the elimination of deprivations. Equality favours the total elimination of deprivations. In fact, all else equal, eliminating deprivations is the ideal with respect to equality; if there were no deprived individuals, there would not be inequality stemming from poverty. Since eliminating deprivations is favoured by equality, it is natural to think that, where we cannot eliminate it, reducing it would be a good second best, from the point of view of equality.

I would like to thank Brad Hooker and Patrick Tomlin for their insightful comments to this paper.

Julia Mosquera

julia.mosquera@iffs.se

1 Philosophy Department, University of Reading, Reading, UK

2 Institute for Futures Studies, Stockholm, Sweden 


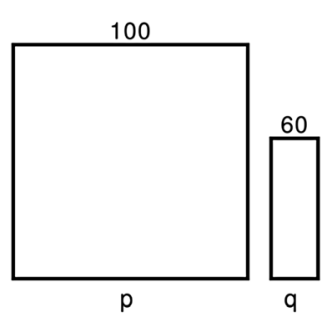

A

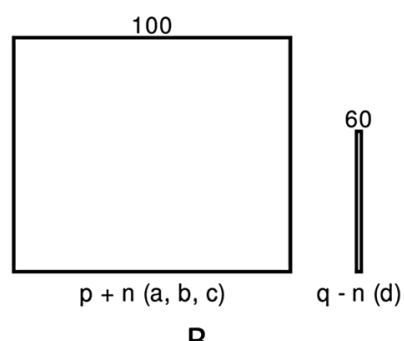

B

Fig. 1 Reduction in the incidence of deprivations. The vertical axis measures the level of wellbeing of the individuals, while the horizontal axis measures the size of the populations. A and B are the same population at different times

Nevertheless, a mere reduction in the incidence of deprivations - as opposed to its elimination-would lead to non-egalitarian outcomes. This paper argues that there is an egalitarian objection to the mere reduction in the incidence of deprivations.

The graphic above represents a reduction in the incidence of deprivation. $B$ results from the drastic reduction of the incidence of deprivation in A. Although both A and B have the same total number of individuals, deprivation is unequally distributed in them. A has a great number of non-deprived individuals who are presumably better-off - all things considered - than a smaller number of non-deprived individuals. In B, on the other hand, the incidence of deprivation is reduced to the point that it is almost inexistent or approaching zero-only a very small number of individuals are affected by deprivations in B Fig. 1.

While A's and B's groups of individuals are at the same level, the size of B's better-off group is much bigger than the group of the worse-off. The pattern of inequality between $\mathrm{A}$ and $\mathrm{B}$ has therefore changed.

Which population is preferable? Both Utility and Priority to the worse-off lead us to the verdict that $\mathrm{B}$ is an improvement on $\mathrm{A}$. For utilitarians, all that matters is the total amount of well-being, here represented graphically as the combined volumes of the two boxes in A versus the combined volumes of the two boxes in B. Since utilitarian accounts of the good do not ascribe moral relevance to the way the good is distributed among individuals, utility cannot make a case against B.

Prioritarianism gives a similar answer with respect to B. B is better than A according to prioritarianism since nobody in $\mathrm{B}$ is worse off than the worse off person in $\mathrm{A}$, and there are more individuals in $\mathrm{B}$ who are better-off.

But does B represent an improvement regarding equality? Both $\mathrm{A}$ and $\mathrm{B}$ are non-ideal situations with respect to equality. But the remaining deprived individuals in B are now comparatively worse-off than the vast majority of the population. Thus, the worse-off people in B are now considerably worse than almost every other member of this world. So, although very few people now have a complaint regarding inequality, the inequality in B seems especially offensive.

It is easier to see this paradoxical conclusion if we first grasp the force of the intuitive argument for reducing the incidence of deprivations:

\section{Egalitarian Argument for Deprivation Reduction}

(1) It is pro tanto bad that some individuals are worse-off than others through no fault of their own-which I will refer to as 'faultless inequality'.

\footnotetext{
${ }^{1}$ There might be other types of inequalities worth considering but in this paper I will solely address the issue of 'faultless inequalities'.
} 
(2) If faultless inequality is pro tanto bad, i.e., (1), reducing faultless inequality is pro tanto good.

(3) Reducing inequality is pro tanto good-modus ponens (1)-(2)

(4) Impoverished individuals are worse-off than non-impoverished individuals through no fault of their own

(5) Reducing deprivation reduces faultless inequality — an implication of (4)

(6) If reducing deprivation reduces faultless inequality, reducing deprivation is pro tanto good-follows from (2) combined with (5).

(7) Reducing the incidence of deprivations is good — modus ponens (5)-(6) - from the point of view of equality.

(1) is the standard formulation of Luck Egalitarianism. ${ }^{2}$ (2) is a conditional claim that stems from (1) and which depends on the assumption that for anything that is pro tanto bad, reducing it is always pro tanto good. ${ }^{3}$ (3) is the result of applying the modus ponens to (1) and (2). (4) is an evaluative claim that assumes that deprived individuals lack access to some elements that are necessary for wellbeing and to which most non-deprived individuals have access. (5) seems to be an implication of (4). (6) follows from the combination of (2) and (5). (7) follows from the combination of (5) and (6) and it collects the thought, possibly common to many people, that a reduction in deprivation is unambiguously good from the point of view of equality.

Paradoxically — and despite the intuitiveness and tightness of the egalitarian argument for deprivation reduction - we have referred to the plausibility of the claim that

(C) a world in which $q$ number of non-deprived individuals exist and $r$ number of deprived individuals exist is not worse with respect to equality — in at least one respect — than a world in which $q+n$ non-deprived individuals exist and $r-n$ deprived individuals exist.

(C) is incompatible with the conclusion of the Egalitarian Argument for Deprivation Reduction (7), and therefore speaks in favor of its rejection. If we are forced to reject (7), this is because at least one of the premises of the egalitarian argument for deprivation reduction is false. Premise (5) - reducing deprivations reduces faultless inequality - seems to be the problematic premise in this argument. The aim of this paper is to show that it is not always the case that the incidence of deprivation increases faultless inequality; or, put it in a different way, there is an egalitarian argument for which reducing the incidence of deprivations does not give rise to more equality since some instances of deprivation reduction are suboptimal from the point of view of equality.

Larry Temkin $(1993,2011)$ devotes extensive discussion to the issue of whether variations in populations size and distribution that do not affect wellbeing can nevertheless modify patterns of inequality. He concludes that they can, but that the way to measure the resulting inequality is not an easy task.

Temkin's work identifies the worry that populations like B - those where the number of worseoff individuals approaches zero, from now on B-type outcomes - are a problem for equality. This makes him question how the inequality of a situation where many are better-off and a few are worseoff compares to another where a few are better-off and many are worse off (Temkin 2011, 240).

Egalitarians may be most offended if just a few are badly-off while the vast majority are well-off, since the inequality then seems particularly gratuitous. (...) those who are

\footnotetext{
${ }^{2}$ See, for example, Cohen (1989). Later on, assumed by others like Richard Arneson, Peter Vallentyne or Larry Temkin. See Richard Arneson (2004)

${ }^{3}$ A clear example of this is the following: Pain is — at least — pro tanto bad. That pain is — at least — pro tanto bad gives us a pro tanto reason to reduce pain.
} 
worse-off are being especially victimized by the situation. (...) it is as if the entire burden of the inequality is borne by those few who are unfortunate enough to be among the worse-off. Given that those few people are much worse off than every other member of their world, it may seem that they have a very large complaint regarding inequality, and correspondingly, that the inequality is especially offensive. (Temkin 2011, 341-2)

After these remarks, Temkin carries on by comparing the egalitarian intuition against B-type outcomes with the intuition that makes most of us reject tyrannical or bullying situations. In a nutshell, Temkin's remarks appeal to the idea that, from an egalitarian point of view, it seems particularly unfair or offensive if a disadvantage, such as poverty for example, is concentrated in a small segment of a population.

But how and whom does this inequality affect? And also, how should this particular inequality be accounted for within the overall inequality of a population This paper aims to explore these questions by providing the measure for an extra dimension of equality that had been either ignored or only partially tackled in the literature. This extra dimension of equality is the one that affects the worse-off individuals in B-type outcomes.

\section{2 (Some of) Equality's Verdicts on B}

Strict Egalitarianism is the view that values equality itself, irrespectively of whether it promotes other values. Larry Temkin's influential work on inequality provides different principles to measure how much inequality a world contains, showing that even within Strict Egalitarianism there are several ways to measure inequality within a population, which all lead to different evaluations with respect to how unequal a distribution is. We will look at those principles in this section and note what their implications for B-type outcomes are.

What should egalitarians, qua egalitarians, say about B-type outcomes? How should they measure the inequality in B? Should inequality be measured in a way that is insensitive to the sizes of the subgroups? If we take the sizes of the groups to be irrelevant, then outcome B does not represent an improvement, and neither does it represent a worsening, in inequality compared with A. Since the amount of wellbeing per person in the better-off and worse-off groups remains constant despite the change in group size, the gap in per person well-being between the members of the worse-off and better-off groups in both A and B is the same. The group size-insensitive comparison would then conclude that they are equally good with respect to equality, which would not support our first intuition with respect to B.

Temkin's measure of inequality is cashed out in terms of 'complaints'. If someone is much better off than I am, the magnitude of my complaint is greater than the magnitude of the other person's complaint. The inequality of a world depends on the quantity and the magnitude of these complaints.

Temkin's relative to the best-off person view of complaints (BOP) (Temkin 1993, 25-6) suggests that

(BOP) The size of someone's complaint depends on how she fares relative to the best-off person.

Applied to our case, BOP does not support our B-type intuition. Since the best-off person in A and the best-off person B are at the same level, and likewise for the worst-off person in A and the worst-off person in $\mathrm{B}$, the difference between the best-off person and the worst-off person 


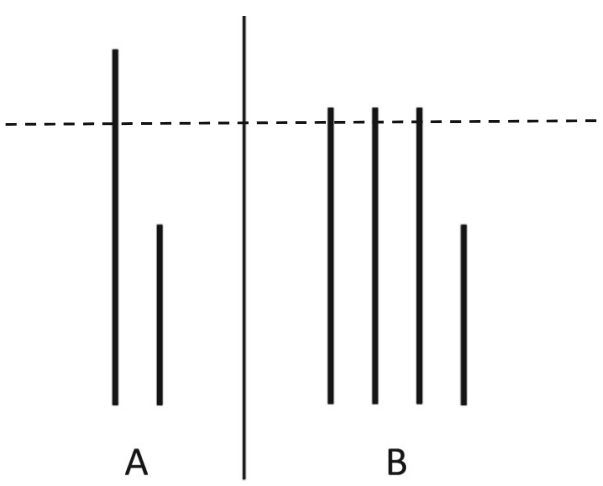

Fig. 2 AVE on the inequality of A and B

will also be the same in A and in B. Thus, BOP implies that the strength of the worst-off's complaint in B is the same size as the strength of the worst-off's complaint in A.

So if we consider BOP in conjunction with the additive principle of complaints, what BOP will conclude is that $\mathrm{B}$ is an improvement on $\mathrm{A}$ in terms of equality because more people have a complaint in A than in B.

The next principle suggested by Temkin to measure inequality complaints is the relative to the average view of complaints (AVE) (Temkin 1993, 25-6). The AVE states that

(AVE) The size of someone's complaint depends on how she fares relative to the average person

If 'average person' refers here to the hypothetical person who is at the arithmetic mean level, various criticisms can be directed towards the AVE. First of all, if all that matters to establish the magnitude of an individual complaint is a comparison with the average utility of that population, AVE will not be able to differentiate between the different situations of the worseoff individuals in the two scenarios described in the graph above (Fig. 2).

Since both the level of the worse-off individuals and the average level are the same for A and B, all that AVE can say about the inequality of A and B here is that the worse-off individual in A and the worse-off individual in B have a complaint of inequality of the same magnitude. But although the average utility of these two populations is the same, and although the worse-off individuals of both populations are at the same level, the inequality that affects the worse-off individuals in both scenarios A and B cannot be the same. After all, the worse off individual in B is worse off than three individuals, while the worse off individual in A is worse off than two individuals.

Secondly, to test the AVE principle more deeply, return to our example with two scenarios - A and B. B's proportion of better- and worse-off individuals resembles the actual scenario of deprivation reduction that our initial graphic showed. B will be compared against A, a scenario of equality equilibrium. And, for the sake of clarity, we will reduce the total size of the populations in both scenarios so that the total number of individuals that compose both scenarios is four. ${ }^{4}$

\footnotetext{
${ }^{4} \mathrm{I}$ acknowledge that this reduction is not a faithful representation of the real scenario of deprivation reduction that this paper is concerned with - the sizes of the group of the deprived and non-deprived individuals are much bigger in the real case. This makes the inequality of this example slightly different from the one of the original example, but I will nevertheless stick to the example for two reasons: firstly, calculating the number of pairwise relations of equality and inequality with real numbers would have been considerably unhelpful in terms of clarity; secondly, the argument about the Standard View and proportional variations in population size that is relevant for this difference is only introduced in section five of this paper.
} 

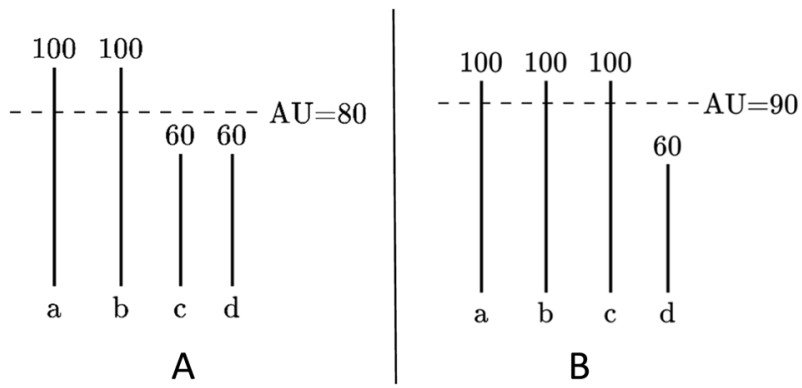

Fig. 3 AVE and the addition of complaints

In the example above, the complaint of individual $d$ in B relative to the mean person would be of a higher magnitude than the complaint of any member of the group of the worse-off in A, compared to the mean person in A (Fig 3).

The previous would be so because.

(B) =90; therefore, d's complaint in B is of magnitude 40 (i.e., 90-30),

whereas,

$(A)=80$; therefore, c's and d's complaint in A are of magnitude 20, respectively (i.e., 80-60).

It seems intuitively true that the complaint of the worse-off individual in B is of a higher magnitude than the complaint of the worse-off individual in A.

But a further problem for AVE has to do with how to aggregate total complaints to evaluate outcomes rather than individual positions, since Temkin's formulation of the view deals only with individual complaints.

If we were to measure the total number of complaints of each of these two scenarios, B would only contain one complaint of magnitude 30, as opposed to two complaints of magnitude 20, each, in scenario A. So, the AVE together with the additive view of complaints would deem $\mathrm{A}$ as more unequal than $\mathrm{B}$.

This result would again clash with our first egalitarian intuition about B-type outcomes. However, there seems to be something particularly disturbing about the inequality that affects the worse off individuals in B. One way to resist BOP's and AVE's verdicts - and so to retain our first intuition that there is something worse about B than A in terms of equality-is by measuring the pairwise inequality of $\mathrm{A}$ and $\mathrm{B}$.

\section{Pairwise Inequality: Aggregative and Distribution-Sensitive}

Another way to account for the amount of inequality in a population is by measuring the number of relations of inequality and the relations of equality ${ }^{5}$ that hold in a population. This can be done by subtracting the number of relations of equality from the number of relations of inequality of each world. From now on we will refer to this way of measuring inequality as aggregative pairwise inequality (API).

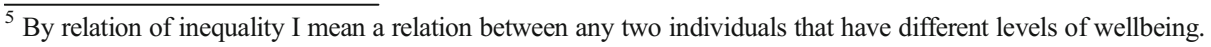
In my example, a relation of inequality will hold between any member of the group of the worse-off and any member of the group of the better-off. By relation of equality, I mean the relation of any individual of the set of the worse-off or better-off with any other individual of its same set. For the purpose of this paper I will ignore the issue of the size of inequalities and assume all relations of inequality are of the same size. I will also assume that only the worse-off individual from a relation of inequality has a complaint about a relation of inequality.
} 
Table 1 Relations of equality and relations of inequality of A, and B

A

$\mathrm{B}$

\begin{tabular}{lll}
\hline Relations of equality & $\mathrm{A}=\{(\mathrm{a}, \mathrm{b}),(\mathrm{c}, \mathrm{d})\}$ & $\mathrm{A}=\{(\mathrm{a}, \mathrm{b}),(\mathrm{a}, \mathrm{c}),(\mathrm{b}, \mathrm{c})\}$ \\
& 2 relations of Equality & 3 relations of Equality \\
Relations of inequality & $\mathrm{B}=\{(\mathrm{a}, \mathrm{c}),(\mathrm{a}, \mathrm{d}),(\mathrm{b}, \mathrm{c}),(\mathrm{b}, \mathrm{d})\}$ & $\mathrm{B}=\{(\mathrm{a}, \mathrm{d}),(\mathrm{b}, \mathrm{d}),(\mathrm{c}, \mathrm{d})\}$ \\
& 4 relations of Inequality & 3 relations of Inequality \\
\hline
\end{tabular}

To test this principle, we will be looking again at the distribution expressed by Fig. 3. In this diagram, $A$ is composed by the individuals $\{a, b, c, d\}$, with $\{a, b\}$ being the members of the group of the better-off, and $\{\mathrm{c}, \mathrm{d}\}$ the members of the group of the worse-off. $\mathrm{B}$, on the hand, is composed by the same four individuals $\{a, b, c, d\}$, but the better-off individuals in $\mathrm{B}$ are $\{\mathrm{a}, \mathrm{b}$, c $\}$, while the worse-off individual is only $\{d\}$.

In $\mathrm{A}$, there are two relations of equality - $\{(\mathrm{a}, \mathrm{b}),(\mathrm{c}, \mathrm{d})\}$ - and four relations of inequality - $\{(a, c),(a, d),(b, c),(b, d)\}$. On the other hand, in B there are three relations of equality$\{(\mathrm{a}, \mathrm{b}),(\mathrm{a}, \mathrm{c}),(\mathrm{b}, \mathrm{c})\}$ - and three relations of inequality $\{(\mathrm{a}, \mathrm{d}),(\mathrm{b}, \mathrm{d}),(\mathrm{c}, \mathrm{d})\}$. The chart above shows these relations (Table 1):

A version of API that only aggregated relations of inequality of a population would conclude that B is better than A. This is so because B possesses three relations of inequality only, while A possesses 4. Thus, this version of API is unable to support our first intuition that there is one respect in which B is worse than A in terms of Equality.

But even if relations of equality become also part of the API's calculus, as Gustaf Arrhenius (2013) suggests, API's verdict about B would still be that B is better than A with respect to Equality. This is so because B contains three relations of equality, as opposed to A which contains only two. The fact that B has one relation of equality more than A would suffice, under some accounts of pairwise inequality, ${ }^{6}$ to make B better than A with respect to equality. In conclusion, a version of API that considers both relations of equality and inequality will determine that B is better than A with respect to equality.

Aggregative accounts such as the API fail to capture the peculiarity of the inequality in B to which we referred at the beginning of this paper. API fails to capture the concentration of relations of inequality that affects the worse-off members of B.

A has fewer relations of equality and more relations of inequality than B. Therefore, B is better, from the point of view of equality, than A, according to API. But there is still something troubling in B-type outcomes. One way to explain this troubling feature is that the API simply aggregates equal and unequal relationships, without looking at how they are distributed. And aggregative principles are famously subject to the separateness of person objection. Perhaps API can be criticised on similar grounds.

The separateness of person objection was originally pressed against utilitarianism by John Rawls, ${ }^{7}$ and later on by Thomas Nagel. ${ }^{8}$ This objection argues that there are boundaries

\footnotetext{
${ }^{6}$ Gustaf Arrhenius suggests this conclusion with his 'Positive Egalitarianism' view. Arrhenius (2013) Especially from $\mathrm{p} 84$.

${ }^{7}$ John Rawls (1999) criticises this aspect of utilitarianism in different passages of A Theory of Justice. One of the features that is common to all of them is that utilitarianism is the idea of an impartial 'sympathetic spectator who conflates all desires into one system'.

${ }^{8}$ Nagel's criterion of individual acceptability is introduced as 'as a way to stay closer to the points of view of the individuals considered', since according to him, simple aggregation 'moves beyond individual points of views'. See Thomas Nagel (1979: 123).
} 
between persons' lives that require a moral separation that aggregative principles do not respect. Utilitarianism holds that, just as present harm to one person can be worth undergoing for the sake of larger benefit to that person later, harm to one person can be justified if necessary either for another person to get a larger benefit or for further people to get a larger combined benefit. The separateness of person objection to utilitarianism is that the multiperson case is not analogous to the single-person case. In the single-person case, present harm is compensated by larger benefit later to the same person. In the multi-person case, harms to some are not compensated by the benefits to others.

Applied to our case, the separateness of person objection suggests that combined gains in equality that result from the aggregation of relations of equality - such as the ones accounted for by API - do not necessarily make the situation for particular individuals better with respect to equality. This means that even when B contains more relations of equality in total, or aggregated interpersonally, that does not imply that the individuals in $\mathrm{B}$ are subject to more equality, too, aggregated intrapersonally. In fact, although A contains more relations of inequality than B, someone in B has more relations of inequality than anyone in A has. This failure to capture the difference between interpersonal and intrapersonal aggregation of relations of inequality makes API insufficient to analyse the particular inequality that affects the worse-off in B-type outcomes.

Temkin's all those better-off view of complaints (ABV) seems closer to our intuition here. Unlike AVE and BOP, ABV contends that the size of one's complaint depends on how she fares relative to all of the others who are better off than she is (Temkin 1993, 25). ABV seems to support our claim that $d$ 's complaints in B are of a greater magnitude than the complaints of any member of the group of the worse-off in $A$. And the fact that almost everybody in $\mathrm{B}$ is better off than $d$ speaks in favor of our intuition that $d$ is particularly badly-off in terms of equality.

But although $\mathrm{ABV}$ is definitely closer to our first intuition expressed at the beginning of our paper by $(\mathrm{C}), \mathrm{ABV}$ does not provide a way to measure the precise amount of badness that constitutes this higher 'magnitude' of the complaint. We might assume that each complaint counts equally. That is, $\mathrm{d}$ has three complaints — one against the inequality with a, one against the inequality with $\mathrm{b}$, and one against the inequality with $\mathrm{c}$. But this still would not vindicate the intuition that there is a particular egalitarian problem with $\mathrm{B}$, because there would still only be three complaints compared to four in A. Thus, the real challenge is to know how we are to precisely measure the inequality of these outcomes.

The following chart illustrate the relations of equality and inequality that affects each individual in both A and B (Table 2):

Table 2 Relations of equality and relations of inequality of each individual of A, and B

\begin{tabular}{cllll}
\hline & $\mathrm{a}$ & $\mathrm{b}$ & $\mathrm{c}$ & $\mathrm{d}$ \\
\hline $\begin{array}{l}\text { Outcome A } \\
\text { Relations of equality }\end{array}$ & $\{(\mathrm{a}, \mathrm{b})\}$ & $\{(\mathrm{a}, \mathrm{b})\}$ & $\{(\mathrm{c}, \mathrm{d})\}$ & $\{(\mathrm{c}, \mathrm{d})\}$ \\
& 1 & 1 & 1 & 1 \\
Relations of inequality & $\{(\mathrm{a}, \mathrm{c}),(\mathrm{a}, \mathrm{d})\}$ & $\{(\mathrm{b}, \mathrm{c}),(\mathrm{b}, \mathrm{d})\}$ & $\{(\mathrm{a}, \mathrm{c}),(\mathrm{b}, \mathrm{c})\}$ & $\{(\mathrm{a}, \mathrm{d}),(\mathrm{b}, \mathrm{d})\}$ \\
& 2 & 2 & 2 & 2 \\
Outcome B & & & & \\
Relations of equality & $\{(\mathrm{a}, \mathrm{b}),(\mathrm{a}, \mathrm{c})\}$ & $\{(\mathrm{a}, \mathrm{b}),(\mathrm{b}, \mathrm{c})\}$ & $\{(\mathrm{a}, \mathrm{c}),(\mathrm{b}, \mathrm{c})\}$ & $\mathbf{0}$ \\
& 2 & 2 & 2 & \\
Relations of inequality & $\{(\mathrm{a}, \mathrm{d})\}$ & $\{(\mathrm{b}, \mathrm{d})\}$ & $\{(\mathrm{c}, \mathrm{d})\}$ & $\{(\mathbf{d}, \mathrm{a}),(\mathbf{d}, \mathrm{b}),(\mathbf{d}, \mathrm{c})\}$ \\
& 1 & 1 & 1 & $\mathbf{3}$ \\
\hline
\end{tabular}


Looking at outcome $B$ in the chart, $d$ is an individual who is not involved in any relation of equality ${ }^{9}$ with any other individual. All the relations $d$ is involved in are relations of inequality. This is so because $d$ is worse off than all of the other members of B. A on the other hand contains more relations of inequality than $B$-four, as opposed to three. But despite this fact, no member of $A$ is subject to the level of inequality that affects one of the members of $B-$ namely $d$. This is so due to various factors:

(i) no individual in $A$ is subject to more than two relations of inequality,

(ii) all of the individuals in $A$ are involved in at least one relation of equality,

(iii) none of the individuals in $A$ is involved in each and every one of the relations of inequality that hold in that world,

(iv) $d$ is not involved in any relation of equality in B,

(v) $d$ is involved in each and every one of the relations of inequality in B.

The previous analysis shows that $d$ is in a particularly bad situation, and therefore makes it salient that we need a pairwise measure of inequality that takes into account also the way relations of inequality are distributed in a world. We will call this principle distributionsensitive pairwise comparison of inequality (DSP).

(DSP) Other things being equal, two populations are equal with respect to equality if they have the same amount of relations of equality and inequality, and these relations are identically distributed among the individuals of each population

According to DSP, it is not only the equal distribution of goods that matters - whether someone has less of a good than others do. In addition, whether someone is subject to more relations of inequality than others, matters, too. This concern introduces a relevant distinction: egalitarians qua egalitarians should also care about second order equality - a notion that refers to equality in the metadistribution of goods, or equality in the distribution of relations of equality and relations of inequality.

DSP is then a function between the number of relations of inequality of a population and how equally or unequally these are distributed among the individuals of that population. It is an improvement with respect to what we have called 'aggregative pairwise comparisons of inequality' in that it suggests a further variable for the measure of inequality; it is not just the amount of relations of inequality or equality that should matter for egalitarians, nor their size, but how these relations of equality and inequality are distributed. One world with the same amount of relations of equality and inequality as another is - not just simply better, but-more egalitarian if no individual has an unequal burden of them.

This 'piling-up' effect captured by DSP reflects the idea of an increasing marginal significance of relations of inequality. Relations of inequality always matter more the more concentrated they are, so it is worse from the point of view of equality when one person suffers two relations of inequality than when two people each suffer one relation of inequality, other things being equal. This is the reason that makes B's inequality feel particularly flagrant. And it is this unfairness that can override the value of one extra relation of equality that $\mathrm{A}$, as opposed to $\mathrm{B}$, contains.

\footnotetext{
${ }^{9}$ Except the relation of being equal to oneself (if we assume the relation of identity as a subtype of the relations of equality).
} 
The graph at the end of the page represents a population of five better off individuals who are at the same level among each other, and five worse off individuals who are at the same level among each other, too Fig. 4.

The effect on equality of reducing the total number of worse off individuals one by one can be represented by a bounded function according to which, every time a worse off individual leaves the group of the worse off, the inequality of the population increases more, which represents the previously mentioned increasing marginal significance of each new relation of inequality.

According to DSE, each new individual that leaves the group of the worse off increases the inequality of the population exponentially in that it makes the distribution of relations of inequality more concentrated. But the interesting phenomenon here is that this increase in inequality stops at the point in which the last worse off individual disappears. At that precise point, the inequality function stops existing since the population achieves perfect equality. This reveals the crucial normative difference between eliminating deprivations and reducing the incidence of these.

So, although making some people better off can make the population better in terms of utility both by raising the number of better-off individuals, or by increasing the number of relations of equality, it can also worsen the inequality of those in the group of the worse-off by increasing the number of the relations of inequality they each have.

We have provided an account, DSP, that is able to measure the inequality that affects the worse-off individuals in B-type outcomes - outcomes where the number of worse-off individuals approaches zero. It is time now to attempt to determine how significant this increasing marginal negative value of the concentration of relations of inequality is. How much should the concentration of relations of inequality count? Should it count indefinitely more than the number of relations of equality in aggregative terms? These issues raise two sets of questions: how much does the piling up effect as opposed to a decrease in relations of inequality matter within Equality? And also, how much does Equality matter in comparison to other values?

When comparing two populations, if those two populations have the same total number of relations of equality and inequality, the concentration of relations of inequality can provide a decisive reason to conclude that a population is worse than another with respect to equality. Nevertheless, the cases in which two populations have the same total amount of relations of equality and inequality are a small minority, and their existence is conditional on both populations having the same number of individuals.

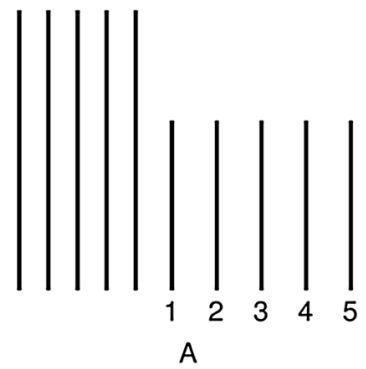

Fig. 4 Progressive reduction of deprivations, up to perfect equality 
For all other cases in which two populations depart from a different number of relations of equality and inequality, the concentration of relations of inequality will become significant at a certain point. We can establish the correlation that the greater the number of better-off individuals and the smaller the number of worse-off individuals is, the worse this concentration will be.

The difficulty is that these scenarios are also scenarios that contain the highest amount of relations of equality due to their greater number of better off individuals, as we have seen. And although egalitarians can prefer populations where the number of relations of equality is greater, egalitarians qua egalitarians should also be concerned about populations where a small minority - the worse-off - are subject to a concentration of relations of inequality.

More research is needed to determine at which precise point this concentration of relations of inequality can in effect outweigh the value provided by this greater amount of relations of equality and therefore to be able to determine that a population is worse-off with respect to Equality.

\section{Conclusion}

Throughout this paper we have seen how, although B-type outcomes - those outcomes where the incidence of deprivation is drastically reduced - do not present a problem for Utility or Priority, they do so for Equality. I have shown how B-type outcomes are a potential problem for equality and have proposed a way to measure the inequality they contain: distribution sensitive pairwise inequality (DSP). I have shown how DSP is more powerful than API to account for the inequality that ultimately affects the worse-off individuals of B-type outcomes because DSP preserves the relational aspect of inequality in both the analysis of inequalities and the analysis of relations of inequality.

I also showed that DSP was sustained in the moral difference between reducing the incidence of deprivations and eliminating them, which has lead us to prove premise (5) of the Argument for deprivation reduction — reducing deprivations reduces inequality - false.

The account of equality sketched in this paper systematizes a new dimension of inequality and is therefore promising in various ways. Firstly, it identifies a new dimension of inequality that will be relevant to many debates of political theory and population. And secondly and more broadly, the political notion of Equality is made stronger with the introduction of an account that can give a precise measure to the basic egalitarian intuition that it matters how many more people are better off than the worst-off. Here we have further confirmation of the well-known idea that the notion of inequality is especially rich and complex, and that there is still a lot of work to do to build the best characterisation of it.

Open Access This article is distributed under the terms of the Creative Commons Attribution 4.0 International License (http:/creativecommons.org/licenses/by/4.0/), which permits unrestricted use, distribution, and reproduction in any medium, provided you give appropriate credit to the original author(s) and the source, provide a link to the Creative Commons license, and indicate if changes were made.

\section{References}

Arneson R (2004) Luck egalitarianism interpreted and defended. Philos Top 32(1):1-20

Arrhenius G (2013) Egalitarian concerns and population ethics. In: Eyal N, Hurst SA, Norheim OF, Wikler D (eds) Inequalities in health. Concepts, measures and ethics. Oxford University Press, Oxford, pp 74-91

Cohen GA (1989) On the currency of egalitarian justice. Ethics 99(4):906-944

Nagel T (1979) Mortal Questions. Cambridge University Press, Cambridge

Rawls J (1971) A Theory of Justice. Harvard University Press, Cambridge, MA 
Temkin L (1993) Inequality. Oxford University Press, Oxford

Temkin L (2011) 'Inequality: a complex, individualistic, and comparative notion'. Philosophical Issues. Social, Political, and Legal Philosophy 11(1):327-353 